Article

\title{
A Renewal Shot Noise Process with Subexponential Shot Marks
}

\section{Yiqing Chen}

College of Business and Public Administration, Drake University, Des Moines, IA 50311, USA;

yiqing.chen@drake.edu

Received: 28 April 2019; Accepted: 3 June 2019; Published: 5 June 2019

Abstract: We investigate a shot noise process with subexponential shot marks occurring at renewal epochs. Our main result is a precise asymptotic formula for its tail probability. In doing so, some recent results regarding sums of randomly weighted subexponential random variables play a crucial role.

Keywords: shot noise; subexponentiality; tail probability; randomly weighted sum; renewal process

\section{Introduction}

Consider a shot noise process $\left\{S_{t}, t \geq 0\right\}$ defined by

$$
S_{t}=\sum_{k=1}^{\infty} X_{k} h\left(t-\tau_{k}\right) 1_{\left(\tau_{k} \leq t\right)}, \quad t \geq 0,
$$

where $X_{1}, X_{2}, \ldots$ are shot marks, which successively occur at times $0 \leq \tau_{1} \leq \tau_{2} \leq \cdots$, and $h(\cdot)$ is a response function non-negative on $[0, \infty)$ and vanishing on $(-\infty, 0)$.

We make the following standard assumptions:

Assumption 1. In the shot noise process (1):

(i) the shot marks $X_{1}, X_{2}, \ldots$ form a sequence of independent and identically distributed (i.i.d.) real-valued random variables with a generic random variable $X$ and distribution $F$;

(ii) their arrival times $\tau_{1}, \tau_{2}, \ldots$ form a sequence of renewal epochs, so that the number of shots by time $t \geq 0$, namely,

$$
N_{t}=\sup \left\{k \in \mathbb{N}: \tau_{k} \leq t\right\},
$$

is an ordinary renewal counting process;

(iii) the two sequences $\left\{X_{1}, X_{2}, \ldots\right\}$ and $\left\{\tau_{1}, \tau_{2}, \ldots\right\}$ are mutually independent;

(iv) the response function $h(\cdot)$ is non-increasing on $[0, \infty)$ with $0<h(0+)<\infty$.

Items (i)-(iii) above equip the shot noise process (1) with a renewal framework. By using the renewal counting process (2), we can rewrite the shot noise process as

$$
S_{t}=\sum_{k=1}^{N_{t}} X_{k} h\left(t-\tau_{k}\right), \quad t \geq 0 .
$$


The assumption $h(0+)>0$ in item (iv) above is to avoid triviality; otherwise, the shot noise process $\left\{S_{t}, t \geq 0\right\}$ will simply take values of $\left\{X_{1}, X_{2}, \ldots\right\}$ at discrete time moments $\left\{\tau_{1}, \tau_{2}, \ldots\right\}$. Also notice that if $h(s) \equiv 1$ for $s \in[0, \infty)$, then the shot noise process reduces to a compound renewal process.

In this paper, we understand the shot noise process (3) in the context of insurance. We interpret the random shot marks as sizes of insurance claims and interpret the response function $h(s)$ as the proportion of a claim to be settled after $s$ units of time have elapsed; that is, each term $X_{k} h\left(t-\tau_{k}\right)$ for $t \geq \tau_{k}$ is the unsettled part at time $t$ of a claim of size $X_{k}$ occurring at time $\tau_{k}$. In this way, $S_{t}$ defined by (3) represents the total amount of unsettled claims at time $t$.

The focus of this work is on the tail probability of the shot noise process $\left\{S_{t}, t \geq 0\right\}$ for the case of heavy-tailed (precisely, subexponential) shot marks. Our result can be applied, e.g., to calculating the solvency capital requirement under contemporary insurance regulatory frameworks such as the Solvency II Directive 2009. As a referee kindly points out, the asymptotic study has an immediate implication for risk management. There is a vast literature devoted to studies that focus on tail probabilities as an essential risk measurement tool; see, e.g., Asmussen et al. (1999); Yang and Wang (2013); Kelly and Jiang (2014); Landsman et al. (2016); Daouia et al. (2018) and Tang et al. (2019).

We end this introduction with a brief literature review of the study of shot noise processes. In the rest of this paper, we present our main result in Section 2, and after preparing two lemmas in Section 3, we show the proof of our main result in Section 4.

\section{A Brief Literature Review}

Shot noise processes were introduced at the beginning of last century, with pioneering works including Campbell (1909) and Schottky (1918). Since then, the topic has been extensively studied and shot noise processes have been used to model a very wide variety of natural phenomena.

Among this huge literature, recent works in the general context of applied probability include Lowen and Teich (1991); Klüppelberg and Mikosch (1995); McCormick (1997); Samorodnitsky (1998); Lund et al. (1999); Brix (1999); Brémaud and Massoulié (2002); Klüppelberg et al. (2003); Møller (2003); Lund et al. (2004); Møller and Torrisi (2005) and Ganesh et al. (2005).

During the recent two decades, there has been another active strand of this literature focusing on applications to insurance and finance; see Basu and Dassios (2002); Dassios and Jang (2003); Jang and Krvavych (2004); Albrecher and Asmussen (2006); Scherer et al. (2012); Jang and Dassios (2013); Weng et al. (2013); Schmidt (2014); Li and Wu (2014) and Liang and Lu (2017).

Most of existing works on this topic model the shot arrivals by a Poisson process or one of its numerous extensions such as a doubly stochastic Poisson process, also known as a Cox process. A literature search only finds a handful of works on renewal shot noise processes, namely Takács (1956); Iksanov (2013); Iksanov et al. (2014) and Dassios et al. (2015). Our current work helps to fill in this gap .

\section{The Main Result}

Throughout this paper, all limit relationships are for $x \rightarrow \infty$ unless stated otherwise. For two positive functions $a(\cdot)$ and $b(\cdot)$, we write $a(x) \sim b(x)$ if $\lim a(x) / b(x)=1$.

A random variable $X$ or its distribution function $F$ with $\bar{F}(x)>0$ for all $x$ is said to be heavy tailed to the right if $E e^{\gamma X}=\infty$ for all $\gamma>0$. One of the most important classes of heavy-tailed distributions is the subexponential class. By definition, a distribution $F$ on $[0, \infty)$ is subexponential, denoted by $F \in \mathcal{S}$, if

$$
\lim _{x \rightarrow \infty} \frac{\overline{F^{n *}}(x)}{\bar{F}(x)}=n
$$


holds for all (or, equivalently, for some) $n=2,3, \ldots$, where $F^{n *}$ denotes the $n$-fold convolution of $F$. More generally, a distribution $F$ on $(-\infty, \infty)$ is still said to be subexponential to the right if $F^{+}(x)=$ $F(x) 1_{(0 \leq x<\infty)}$ is subexponential. The class $\mathcal{S}$ is very broad in the sense that it covers distributions with very different heavy tails ranging from very heavy (such as the Pareto distribution), moderately heavy (such as the lognormal distribution), and mildly heavy (such as the Weibull distribution). The famous class $\mathcal{R}$, as a subclass of $\mathcal{S}$, covers very heavy-tailed distributions. By definition, for a distribution function $F$ on $\mathbb{R}$, we write $F \in \mathcal{R}_{-\alpha}$ for some $0<\alpha<\infty$ if its right tail is regularly varying with index $-\alpha$, that is,

$$
\lim _{x \rightarrow \infty} \frac{\bar{F}(x y)}{\bar{F}(x)}=y^{-\alpha}, \quad y>0 .
$$

The reader is referred to Bingham et al. (1987); Resnick (1987); Embrechts et al. (1997) and Foss et al. (2011) for textbook treatments of heavy-tailed distributions with applications to insurance and finance.

Now we are ready to state our main result.

Theorem 1. Consider the shot noise process (3). If $F$ is subexponential, then for any $t>0$ such that $P\left(\tau_{1} \leq t\right)>0$, we have

$$
P\left(S_{t}>x\right) \sim \int_{0}^{t} P(X h(t-s)>x) d E N_{s} .
$$

Some immediate refinements of Theorem 1 follow. First, if $\left\{N_{t}, t \geq 0\right\}$ is a homogeneous Poisson process with rate $\lambda>0$, then plugging in $E N_{S}=\lambda s$ into (4) yields

$$
P\left(S_{t}>x\right) \sim \lambda \int_{0}^{t} P(X h(s)>x) d s .
$$

This corresponds to Theorem 2.1 of Tang (2006) with $\gamma=0$.

Second, if $F \in \mathcal{R}_{-\alpha}$ for some $0<\alpha<\infty$, then subject to a standard argument based on Potter's bounds (see Proposition 2.2.3 of Bingham et al. (1987)), the asymptotic formula (4) becomes

$$
\lim _{x \rightarrow \infty} \frac{P\left(S_{t}>x\right)}{\bar{F}(x)}=\int_{0}^{t} h^{\alpha}(t-s) d E N_{s} .
$$

Actually, by Assumption 1(iv), the response function $h(\cdot)$ is non-increasing on $[0, \infty)$ and bounded by $0<h(0+)<\infty$. Thus, as $x$ becomes large, so does $x / h(t-s)$ for all $s \in(0, t]$, where $x / 0$ is understood as $\infty$ by convention. For arbitrarily fixed small $\varepsilon>0$, by Potter's bounds, it holds for all large $x$ and all $s \in(0, t]$ that

$$
\begin{aligned}
& (1-\varepsilon) \min \left\{h^{\alpha+\varepsilon}(t-s), h^{\alpha-\varepsilon}(t-s)\right\} \\
\leq & \frac{P(X h(t-s)>x)}{\bar{F}(x)} \\
\leq & (1+\varepsilon) \max \left\{h^{\alpha+\varepsilon}(t-s), h^{\alpha-\varepsilon}(t-s)\right\} .
\end{aligned}
$$


Plugging these bounds into the right-hand side of (4) yields

$$
\begin{aligned}
& (1-\varepsilon) \int_{0}^{t} \min \left\{h^{\alpha+\varepsilon}(t-s), h^{\alpha-\varepsilon}(t-s)\right\} d E N_{s} \\
\leq & \frac{P\left(S_{t}>x\right)}{\bar{F}(x)} \\
\leq & (1+\varepsilon) \int_{0}^{t} \max \left\{h^{\alpha+\varepsilon}(t-s), h^{\alpha-\varepsilon}(t-s)\right\} d E N_{s} .
\end{aligned}
$$

Letting $\varepsilon \downarrow 0$, by the dominated convergence theorem, the two bounds above coincide with each other and equal to $\int_{0}^{t} h^{\alpha}(t-s) d E N_{s}$. This verifies (5).

If, moreover, $\left\{N_{t}, t \geq 0\right\}$ is a homogeneous Poisson process with rate $\lambda>0$, then the asymptotic formula (5) is further refined to

$$
\lim _{x \rightarrow \infty} \frac{P\left(S_{t}>x\right)}{\bar{F}(x)}=\lambda \int_{0}^{t} h^{\alpha}(s) d s
$$

\section{Lemmas}

This section recalls two important lemmas regarding the tail probabilities of sums of randomly weighted subexponential random variables. The following first lemma is a restatement of Theorem 1 of Tang and Yuan (2014):

Lemma 1. Let $X_{1}, \ldots, X_{n}$ be $n$ i.i.d. random variables with common distribution function $F \in \mathcal{S}$, and let $\theta_{1}, \ldots, \theta_{n}$ be $n$ non-negative, bounded, and not-degenerate-at-zero random variables independent of $X_{1}, \ldots, X_{n}$. Then

$$
P\left(\sum_{k=1}^{n} \theta_{k} X_{k}>x\right) \sim \sum_{k=1}^{n} P\left(\theta_{k} X_{k}>x\right) .
$$

The next lemma, attributed to a recent work of the author in Chen (2019), establishes a Kesten-type upper bound for the tail probabilities of the sums of randomly weighted subexponential random variables:

Lemma 2. Let $\left\{X_{1}, X_{2}, \ldots\right\}$ be a sequence of i.i.d. and real-valued random variables with common distribution function $F \in \mathcal{S}$, let $\left\{\theta_{1}, \theta_{2}, \ldots\right\}$ be another sequence of non-negative and uniformly bounded random variables independent of $\left\{X_{1}, X_{2}, \ldots\right\}$. Then for any $\varepsilon>0$, there exists a constant $C_{\varepsilon}>0$ such that

$$
P\left(\sum_{k=1}^{n} \theta_{k} X_{k}>x\right) \leq C_{\varepsilon}(1+\varepsilon)^{n} \sum_{k=1}^{n} P\left(\theta_{k} X_{k}>x\right)
$$

holds for all $n \in \mathbb{N}$ and all $x \geq 0$.

\section{Proof of Theorem 1}

For an arbitrarily fixed $M \in \mathbb{N}$, we expand the tail probability of $S_{t}$ as

$$
\begin{aligned}
P\left(S_{t}>x\right) & =\left(\sum_{n=1}^{M}+\sum_{n=M+1}^{\infty}\right) P\left(\sum_{k=1}^{n} X_{k} h\left(t-\tau_{k}\right)>x, N_{t}=n\right) \\
& =I_{1}+I_{2} .
\end{aligned}
$$


We apply Lemma 1 to deal with $I_{1}$. For each $n=1, \ldots, M$, we condition each tail probability

$$
P\left(\sum_{k=1}^{n} X_{k} h\left(t-\tau_{k}\right)>x, N_{t}=n\right)
$$

in $I_{1}$ on $\left(N_{t}=n\right)$ and then interpret each conditional random variable $h\left(t-\tau_{k}\right) \mid\left(N_{t}=n\right)$ as a random weight $\theta_{k}$. To be strict, such a random variable $\theta_{k}$ involves both arguments $t$ and $n$, but this does not matter; key requirements for the applicability of Lemma 1 are that the random weight $\theta_{1}, \ldots, \theta_{n}$ are bounded and independent of the shot marks $X_{1}, \ldots, X_{n}$. Thus, by Lemma 1 we obtain

$$
P\left(\sum_{k=1}^{n} X_{k} h\left(t-\tau_{k}\right)>x, N_{t}=n\right) \sim \sum_{k=1}^{n} P\left(X_{k} h\left(t-\tau_{k}\right)>x, N_{t}=n\right) .
$$

It follows that

$$
\begin{aligned}
I_{1} & \sim \sum_{n=1}^{M} \sum_{k=1}^{n} P\left(X_{k} h\left(t-\tau_{k}\right)>x, N_{t}=n\right) \\
& =\left(\sum_{n=1}^{\infty} \sum_{k=1}^{n}-\sum_{n=M+1}^{\infty} \sum_{k=1}^{n}\right) P\left(X_{k} h\left(t-\tau_{k}\right)>x, N_{t}=n\right) \\
& =I_{11}+I_{12} .
\end{aligned}
$$

By interchanging the order of the two sums in $I_{11}$, we have

$$
\begin{aligned}
I_{11} & =\sum_{k=1}^{\infty} \sum_{n=k}^{\infty} P\left(X_{k} h\left(t-\tau_{k}\right)>x, N_{t}=n\right) \\
& =\sum_{k=1}^{\infty} P\left(X_{k} h\left(t-\tau_{k}\right)>x, N_{t} \geq k\right) \\
& =\sum_{k=1}^{\infty} P\left(X_{k} h\left(t-\tau_{k}\right)>x, \tau_{k} \leq t\right) \\
& =\sum_{k=1}^{\infty} \int_{0}^{t} P\left(X_{k} h(t-s)>x\right) P\left(\tau_{k} \in d s\right) \\
& =\int_{0}^{t} P(X h(t-s)>x) d E N_{s},
\end{aligned}
$$

where the last step is due to $\sum_{k=1}^{\infty} P\left(\tau_{k} \leq s\right)=E N_{s}$. For $I_{12}$, by the monotonicity of the response function $h(\cdot)$, we derive

$$
\begin{aligned}
I_{12} & \leq \sum_{n=M+1}^{\infty} \sum_{k=1}^{n} P\left(X_{k} h\left(t-\tau_{1}\right)>x, N_{t}=n\right) \\
& =\sum_{n=M+1}^{\infty} n \int_{0}^{t} P(X h(t-s)>x) P\left(N_{t-s}=n-1\right) P\left(\tau_{1} \in d s\right) \\
& =\int_{0}^{t} P(X h(t-s)>x) E\left[\left(N_{t-s}+1\right) 1_{\left(N_{t-s} \geq M\right)}\right] P\left(\tau_{1} \in d s\right) \\
& \leq E\left[\left(N_{t}+1\right) 1_{\left(N_{t} \geq M\right)}\right] \int_{0}^{t} P(X h(t-s)>x) P\left(\tau_{1} \in d s\right) .
\end{aligned}
$$


Thus, for arbitrarily fixed $\delta>0$, due to $E\left[N_{t}\right]<\infty$, we can choose $M$ large enough such that

$$
E\left[\left(N_{t}+1\right) 1_{\left(N_{t} \geq M\right)}\right] \leq \delta .
$$

It follows that

$$
I_{12} \leq \delta \int_{0}^{t} P(X h(t-s)>x) P\left(\tau_{1} \in d s\right) \leq \delta \int_{0}^{t} P(X h(t-s)>x) d E N_{s} .
$$

We apply Lemma 2 to deal with $I_{2}$ in (6). As in dealing with $I_{1}$, for each $n \geq M+1$ we condition each tail probability

$$
P\left(\sum_{k=1}^{n} X_{k} h\left(t-\tau_{k}\right)>x, N_{t}=n\right)
$$

in $I_{2}$ on $\left(N_{t}=n\right)$ and then interpret each conditional random variable $h\left(t-\tau_{k}\right) \mid\left(N_{t}=n\right)$ as a random weight $\theta_{k}$. As explained above, these random weights $\theta_{1}, \theta_{2}, \ldots$, though involving both arguments $t$ and $n$, are uniformly bounded and independent of the shot marks $X_{1}, X_{2}, \ldots$ This justifies the applicability of Lemma 2. Thus, for any $\varepsilon>0$, there exists a constant $C_{\varepsilon}>0$ such that, for all $n \in \mathbb{N}$ and $x \geq 0$,

$$
P\left(\sum_{k=1}^{n} X_{k} h\left(t-\tau_{k}\right)>x, N_{t}=n\right) \leq C_{\varepsilon}(1+\varepsilon)^{n} \sum_{k=1}^{n} P\left(X_{k} h\left(t-\tau_{k}\right)>x, N_{t}=n\right) .
$$

It follows that

$$
\begin{aligned}
I_{2} & \leq C_{\varepsilon} \sum_{n=M+1}^{\infty}(1+\varepsilon)^{n} \sum_{k=1}^{n} P\left(X_{k} h\left(t-\tau_{k}\right)>x, N_{t}=n\right) \\
& \leq C_{\varepsilon} \sum_{n=M+1}^{\infty}(1+\varepsilon)^{n} n P\left(X h\left(t-\tau_{1}\right)>x, N_{t}=n\right) \\
& =C_{\varepsilon} \sum_{n=M+1}^{\infty}(1+\varepsilon)^{n} n \int_{0}^{t} P(X h(t-s)>x) P\left(N_{t-s}=n-1\right) P\left(\tau_{1} \in d s\right) \\
& =C_{\varepsilon} \int_{0}^{t} P(X h(t-s)>x) E\left[(1+\varepsilon)^{N_{t-s}+1}\left(N_{t-s}+1\right) 1_{\left(N_{t-s} \geq M\right)}\right] P\left(\tau_{1} \in d s\right) \\
& \leq C_{\varepsilon} E\left[(1+\varepsilon)^{N_{t}+1}\left(N_{t}+1\right) 1_{\left(N_{t} \geq M\right)}\right] \int_{0}^{t} P(X h(t-s)>x) P\left(\tau_{1} \in d s\right) .
\end{aligned}
$$

It is easy to verify that for a general renewal counting process $\left\{N_{t}, t \geq 0\right\}$, regardless of $E \tau_{1}<\infty$, there is always $b>1$ such that $E b^{N_{t}}<\infty$; see also Theorem 1 of Kočetova et al. (2009). Thus, for arbitrarily fixed $\delta>0$, we can choose some small $\varepsilon>0$ and large $M>0$ such that

$$
C_{\varepsilon} E\left[(1+\varepsilon)^{N_{t}+1}\left(N_{t}+1\right) 1_{\left(N_{t} \geq M\right)}\right] \leq \delta .
$$

It follows that

$$
I_{2} \leq \delta \int_{0}^{t} P(X h(t-s)>x) P\left(\tau_{1} \in d s\right) \leq \delta \int_{0}^{t} P(X h(t-s)>x) d E N_{s} .
$$

Finally, simply combining (6)-(10) together and making use of the arbitrariness of $\delta>0$, we prove the asymptotic relation (4). 
Funding: This research was supported by a Centers of Actuarial Excellence (CAE) Research Grant (2018-2021) from the Society of Actuaries (SOA).

Conflicts of Interest: The author declares no conflict of interest.

\section{References}

Albrecher, Hansjörg, and Søren Asmussen. 2006. Ruin probabilities and aggregate claims distributions for shot noise Cox processes. Scandinavian Actuarial Journal 2: 86-110. [CrossRef]

Asmussen, Søren, Hanspeter Schmidli, and Volker Schmidt. 1999. Tail probabilities for non-standard risk and queueing processes with subexponential jumps. Advances in Applied Probability 31: 422-47. [CrossRef]

Basu, Sankarshan, and Angelos Dassios. 2002. A Cox process with log-normal intensity. Insurance: Mathematics and Economics 31: 297-302. [CrossRef]

Bingham, Nicholas H., Chrles M. Goldie, and Jozef L. Teugels. 1987. Regular Variation. Cambridge: Cambridge University Press.

Brémaud, Pierre, and Laurent Massoulié. 2002. Power spectra of general shot noises and Hawkes point processes with a random excitation. Advances in Applied Probability 34: 205-22. [CrossRef]

Brix, Anders. 1999. Generalized gamma measures and shot-noise Cox processes. Advances in Applied Probability 31 : 929-53. [CrossRef]

Campbell, Norman. 1909. The study of discontinuous phenomena. Proceedings of the Cambridge Philosophical Society 15 : 117-36.

Chen, Yiqing. 2019. A Kesten-type bound for sums of randomly weighted subexponential random variables. Statistics $\mathcal{E}$ Probability Letters, to appear.

Daouia, Abdelaati, Stéphane Girard, and Gilles Stupfler. 2018. Estimation of tail risk based on extreme expectiles. Journal of the Royal Statistical Society: Series B (Statistical Methodology) 80: 263-92. [CrossRef]

Dassios, Angelos, and Ji-Wook Jang. 2003. Pricing of catastrophe reinsurance and derivatives using the Cox process with shot noise intensity. Finance and Stochastics 7: 73-95. [CrossRef]

Dassios, Angelos, Jiwook Jang, and Hongbiao Zhao. 2015. A risk model with renewal shot-noise Cox process. Insurance: Mathematics and Economics 65: 55-65. [CrossRef]

Embrechts, Paul, Claudia Klüppelberg, and Thomas Mikosch. 1997. Modelling Extremal Events: For Insurance and Finance. Berlin/Heidelberg: Springer Science \& Business Media.

Foss, Sergey, Dmitry Korshunov, and Stan Zachary. 2011. An Introduction to Heavy-tailed and Subexponential Distributions. New York: Springer.

Ganesh, Ayalvadi, Claudio Macci, and Giovanni Torrisi. 2005. Sample path large deviations principles for Poisson shot noise processes and applications. Electronic Journal of Probability 10: 1026-43. [CrossRef]

Iksanov, Alexander. 2013. Functional limit theorems for renewal shot noise processes with increasing response functions. Stochastic Processes and their Applications 123: 1987-2010. [CrossRef]

Iksanov, Alexander, Alexander Marynych, and Matthias Meiners. 2014. Limit theorems for renewal shot noise processes with eventually decreasing response functions. Stochastic Processes and their Applications 124: $2132-70$. [CrossRef]

Jang, Jiwook, and Angelos Dassios. 2013. A bivariate shot noise self-exciting process for insurance. Insurance: Mathematics and Economics 53: 524-32. [CrossRef]

Jang, Ji-Wook, and Yuriy Krvavych. 2004. Arbitrage-free premium calculation for extreme losses using the shot noise process and the Esscher transform. Insurance: Mathematics and Economics 35: 97-111. [CrossRef]

Kelly, Bryan, and Hao Jiang. 2014. Tail risk and asset prices. The Review of Financial Studies 27: 2841-71. [CrossRef]

Klüppelberg, Claudia, and Thomas Mikosch. 1995. Explosive Poisson shot noise processes with applications to risk reserves. Bernoulli 1: 125-47. [CrossRef]

Klüppelberg, Claudia, Thomas Mikosch, and Anette Schärf. 2003. Regular variation in the mean and stable limits for Poisson shot noise. Bernoulli 9: 467-96. [CrossRef] 
Kočetova, Jelena, Remigijus Leipus, and Jonas Šiaulys. 2009. A property of the renewal counting process with application to the finite-time ruin probability. Lithuanian Mathematical Journal 49: 55-61. [CrossRef]

Landsman, Zinoviy, Udi Makov, and Tomer Shushi. 2016. Tail conditional moments for elliptical and log-elliptical distributions. Insurance: Mathematics and Economics 71: 179-88. [CrossRef]

Liang, Xiaoqing, and Yi Lu. 2017. Indifference pricing of a life insurance portfolio with risky asset driven by a shot-noise process. Insurance: Mathematics and Economics 77: 119-32. [CrossRef]

Li, Xiaohu, and Jintang Wu. 2014. Asymptotic tail behavior of Poisson shot-noise processes with interdependence between shock and arrival time. Statistics \& Probability Letters 88: 15-26.

Lowen, Steven B., and Malvin C. Teich. 1991. Doubly stochastic Poisson point process driven by fractal shot noise. Physical Review A 43: 4192-215. [CrossRef]

Lund, Robert B., Ronald W. Butler, and Robert L. Paige. 1999. Prediction of shot noise. Journal of Applied Probability 36: 374-88. [CrossRef]

Lund, Robert, William P. McCormick, and Yuanhui Xiao. 2004. Limiting properties of Poisson shot noise processes. Journal of Applied Probability 41: 911-18. [CrossRef]

McCormick, William P. 1997. Extremes for shot noise processes with heavy tailed amplitudes. Journal of Applied Probability 34: 643-56. [CrossRef]

Møller, Jesper. 2003. Shot noise Cox processes. Advances in Applied Probability 35: 614-40. [CrossRef]

Møller, Jesper, and Giovanni Luca Torrisi. 2005. Generalised shot noise Cox processes. Advances in Applied Probability 37: 48-74. [CrossRef]

Resnick, Sidney I. 1987. Extreme Values, Regular Variation and Point Processes. New York: Springer.

Samorodnitsky, Gennady. 1998. Tail behavior of some shot noise processes. In A Practical Guide to Heavy Tails: Statistical Techniques and Applications. Boston: Birkhäuser Boston, Inc., pp. 473-86.

Scherer, Matthias, Ludwig Schmid, and Thorsten Schmidt. 2012. Shot-noise driven multivariate default models. European Actuarial Journal 2: 161-86. [CrossRef]

Schmidt, Thorsten. 2014. Catastrophe insurance modeled by shot-noise processes. Risks 2: 3-24. [CrossRef]

Schottky, Walter. 1918. Über spontane Stromschwankungen in verschiedenen Elektrizitätsleitern. Annalen der physik 362: 541-67. [CrossRef]

Takács, Lajos. 1956. On secondary stochastic processes generated by recurrent processes. Acta Mathematica Hungarica 7: 17-29. [CrossRef]

Tang, Qihe. 2006. On convolution equivalence with applications. Bernoulli 12: 535-49. [CrossRef]

Tang, Qihe, Zhaofeng Tang, and Yang Yang. 2019. Sharp asymptotics for large portfolio losses under extreme risks. European Journal of Operational Research 276: 710-22. [CrossRef]

Tang, Qihe, and Zhongyi Yuan. 2014. Randomly weighted sums of subexponential random variables with application to capital allocation. Extremes 17: 467-93. [CrossRef]

Weng, Chengguo, Yi Zhang, and Ken Seng Tan. 2013. Tail behavior of Poisson shot noise processes under heavy-tailed shocks and Actuarial applications. Methodology and Computing in Applied Probability 15: 655-82. [CrossRef]

Yang, Yang, and Yuebao Wang. 2013. Tail behavior of the product of two dependent random variables with applications to risk theory. Extremes 16: 55-74. [CrossRef]

(C) 2019 by the author. Licensee MDPI, Basel, Switzerland. This article is an open access article distributed under the terms and conditions of the Creative Commons Attribution (CC BY) license (http:/ / creativecommons.org/licenses/by/4.0/). 